\title{
Meningococcal Meningitis Data Analysis, Amhara National Regional State of Ethiopia, 2015-2019; a Descriptive Cross-Sectional Study
}

\author{
Tesfahun Abye Meshesha", Kefyalew Amene, Tigist Abera, Rozina Tariku \\ Public Health Emergency Management; Ethiopian Public Health Institute, Addis Ababa, Ethiopia \\ Email address: \\ Tesfahunabye1900@gmail.com (T. A. Meshesha) \\ ${ }^{*}$ Corresponding author \\ To cite this article: \\ Tesfahun Abye Meshesha, Kefyalew Amene, Tigist Abera, Rozina Tariku. Meningococcal Meningitis Data Analysis, Amhara National \\ Regional State of Ethiopia, 2015-2019; a Descriptive cross-Sectional Study. Science Journal of Clinical Medicine. \\ Vol. 10, No. 1, 2021, pp. 1-6. doi: 10.11648/j.sjcm.20211001.11
}

Received: January 3, 2021; Accepted: January 16, 2021; Published: January 30, 2021

\begin{abstract}
Background: Meningococcal meningitis is a disease of major public health importance especially for countries in the meningitis belt. The retrospective data analysis had provided helpful information to understand the current prevalence of meningococcal meningitis. The trends of meningococcal meningitis had provided useful estimates on the effect of seasonal variability of meningitis, and the distribution of disease burden. So the objective of this study is to assess and describes the magnitude and distribution of Meningitis in Amhara national regional state of Ethiopia from 2015- 2019. Method: Meningococcal meningitis surveillance data of the Amhara region from 2015-2019 were reviewed to describe the disease epidemiology. The study involves a retrospective descriptive analysis of collected clinical data and line list reported to EPHI from 2015-2019 through the Public Health Emergency Management Surveillance system. Result: Of the total 894 patients and 25 deaths, highly contributing zones were North Shewa with 195 (21.81\%) patients, North Gondar with 145 (16.22\%) patients, and South Wollo with 101(11.30\%) patients. Of the total patients, 534 were treated as impatient patients while 371 were treated as outpatient patients. The regional patient fatality rate is 2.80 and the attack rate is 4.59 per 100,000 population. Meningococcal meningitis morbidity is high in North Gondar, South Wollo, and North Shewa, and mortality are high in North Shewa, South Wollo, and East Gojjam respectively. There was high morbidity with low mortality like in North Gondar and North Shewa. Conclusion: Meningococcal meningitis is affected by geographical factors common in arid areas. In epidemic week 38 of 2015 (dry season), there was a meningococcal meningitis outbreak in north Gondar specifically in west Armachiho district. Patient management is poor in those high mortality areas like North Shewa, South Wollo, and East Gojjam. Laboratory-based surveillance should be implemented to identify common serotypes of N. meningitides.
\end{abstract}

Keywords: Meningococcal Meningitis, Outbreak, Meningitis Belt, Surveillance

\section{Introduction}

Neisseria meningitides is a leading causative agent of bacterial meningitis and septicemia, particularly in children $<5$ years old and young adults, and is estimated to cause 500,000 patients and 50,000 deaths globally each year [1]. In non-epidemic conditions, only laboratory investigation of cerebrospinal fluid (CSF), obtained by lumbar puncture, can reliably differentiate meningococcal meningitis from other types of bacterial meningitis. Meningococcal meningitis is a disease of major public health importance especially for countries in the meningitis belt [2]. Meningococcal meningitis is one of the most severe infectious diseases, not only causing physical and neurologic sequelae, but also continues to be an important cause of mortality [3]. Acute bacterial meningitis is most commonly caused by Neisseria meningitides, Streptococcus pneumoniae and Homophiles influenza Type b $[4,5]$. The incidence of meningococcal diseases is generally low since the advent of national meningococcal vaccination programs. However, a much higher incidence (up to 1000 patients per 100,000 people) has been observed during epidemics, particularly in countries in the African meningitis belt [6]. 
PCVs are a remarkable public health success as they induce herd immunity and are associated with reductions in invasive pneumococcal disease globally [7].

Ethiopia had regularly affected by both the endemic and epidemic forms of the disease. The epidemic season for meningitis coincides with the dry season and ends with the arrival of the African rainy season [8]. The most recent major outbreak affecting the whole country occurred in 1988-1989, with nearly 50000 patients and 990 deaths, and an overall attack rate of 133 per 100000 [9]. Amhara national regional state had experienced an increased number of patients reported in 2000 [10, 11]. Despite a strong seasonality, the determinants of meningitis dynamics are still poorly understood [12]. Trends of Meningitis in the country may indicate the current status of the disease and the risk factors related to it. Amhara national regional state is located on the African meningitis belt and bordering with meningitis prone countries [13]. The recent trends of bacterial meningitis had provided useful estimates on effect of seasonal variability of meningitis and geographical distribution of diseases. The retrospective data analysis had provided helpful information to understand the current prevalence of Meningococcal meningitis in study area and would help in the strengthening of Meningococcal meningitis control [14]. I therefore reviewed meningococcal meningitis surveillance data of Amhara region from 2015-2019 to describe the disease epidemiology. The general objective of the analysis is to asses and describe the magnitude and distribution of Meningitis in Amhara national regional state of Ethiopia from 2015- 2019.

\section{Materials and Methods}

\subsection{Study Area}

Ethiopia is administratively sub-divided into nine regional states and two city administrations. Amhara national regional state is one of the nine administrative regions of Ethiopia. According to the 2010 Health and Health Related Indicators published by FMoH, Amhara national regional state has 17 Hospitals, 520 Health Centers and 2,941 Health Posts. Based on 2010 figures from the Central Statistical Agency (CSA) of Ethiopia, the Amhara national regional state has an estimated total population of 18,167,982 consisting of 9,110,481 male and 9,057,501 female. $87.4 \%$ of the population is estimated to be rural inhabitants, while $12.6 \%$ are urban dwellers [15]. The region has an estimated area of $154,708.96$ square kilometers, this region has an estimated density of 117.4 people per square kilometer. The regional meningitis surveillance data is collected from all health facilities excluding health posts (as health professionals may not diagnose the meningococcal meningitis diseases properly) in different zones and districts of the region.

\subsection{Study Design and Period}

The study involves a retrospective descriptive analysis of clinical and laboratory data collected by weekly and line list reporting forms from 2015-2019 reported to EPHI through the public Health Emergency Management Surveillance system.

\subsection{Study Population}

The study included all the suspected and confirmed meningococcal meningitis patients reported during 20152019 from Amhara national regional health bureau offered to EPHI.

\subsection{Limitation of Study}

Since the study was based on secondary data the quality of the data can't definitely be assured. Under reporting and incomplete of variables was observed and may not show the overall burden of patients in the study areas. Health posts and many of health centers didn't have a laboratory capacity to diagnose the disease which may hide the burden of disease.

\subsection{Variables}

The variable includes the total Meningitis, out patients, in patients (wheatear clinical or confirmed patients), Deaths, and Place and also Week, Months, and Year (Time) were the variables.

\subsection{Analysis and Data Management}

Calculation of relevant quantitative measures were conducted by excel to identify any outbreaks, differences with zones and districts and the Meningitis patients related to seasons in the study area. Tried to ensure to have the right data records and performing quality control checks on each data field. Graphs and tables used to show the findings

\section{Result}

\subsection{Analysis by Place}

A total of 894 meningococcal meningitis patients and 25 deaths were reported from Amhara national regional state to Ethiopian Public Health Institute through national Public Health Emergency Management center, within the five years study period (2015- 2019). Of the total patients, highly contributing zones were North Shewa with 195 (21.81\%) patients, North Gondar with 145 (16.22\%) patients, South Wollo with 101(11.30\%) patients and Gondar town with 99 $(11.00 \%)$ patients. The rest $(40 \%)$ were reported from other zones of the region. Except Argoba special district all zones of the region reported meningitis patients and Desse town occasionally reported only 6 patients throughout the whole 5 year study period.

Of the total regional patients, 534 patients were treated as impatient patients while 371 were treated as outpatient patients. But when we see at zonal level south Gondar, North Shewa, South wollo, East Gojjam and Gondar town treats more than $80 \%$ of meningitis patients as impatient while the rest zones treats patients at outpatient department. Awi zone, north Gondar and Waghimra zones reports most of meningococcal meningitis patients from outpatient departmet. 


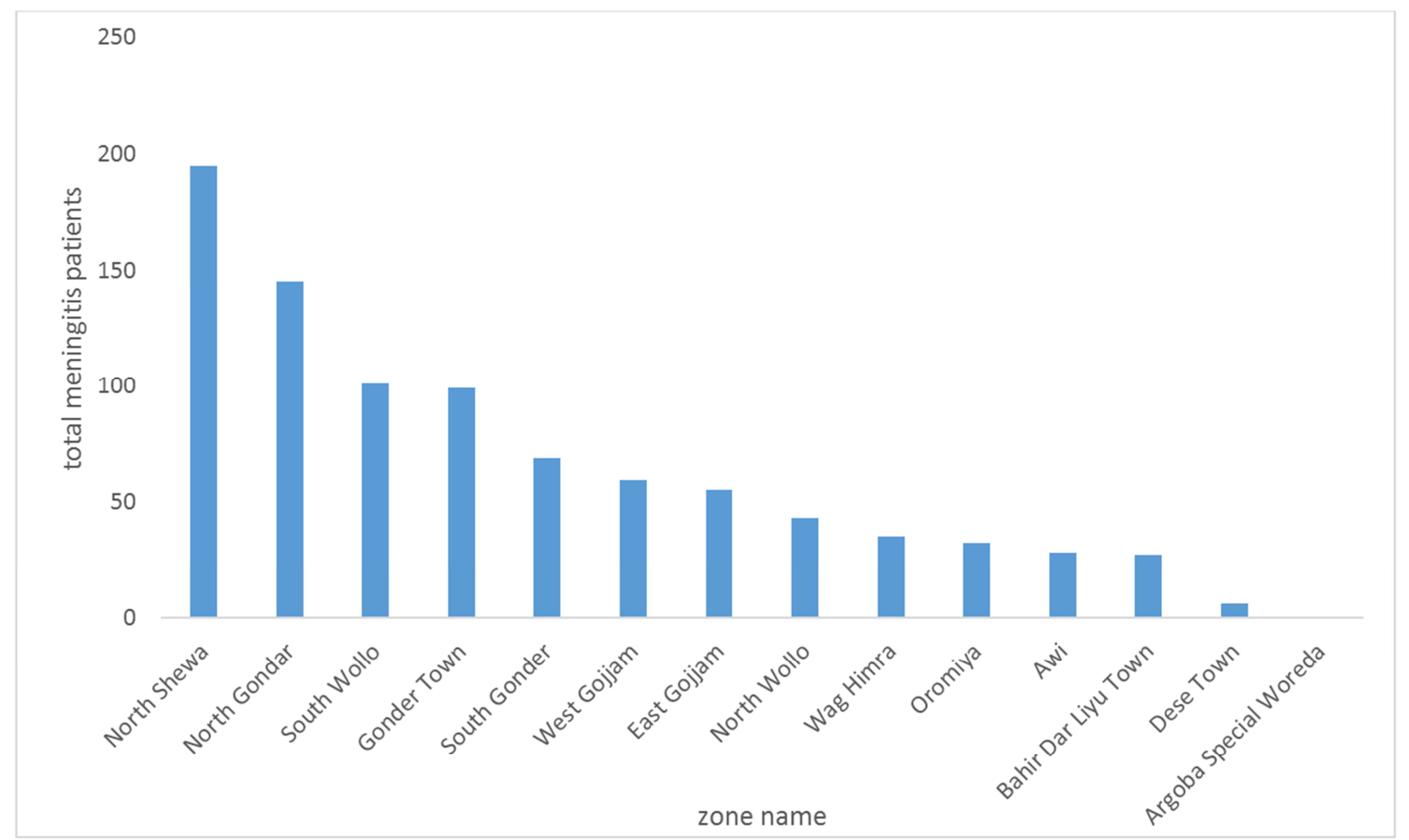

Figure 1. Total Meningitis Patient Distribution by zones of Amhara national regional state, Ethiopia, 2015- 2019.

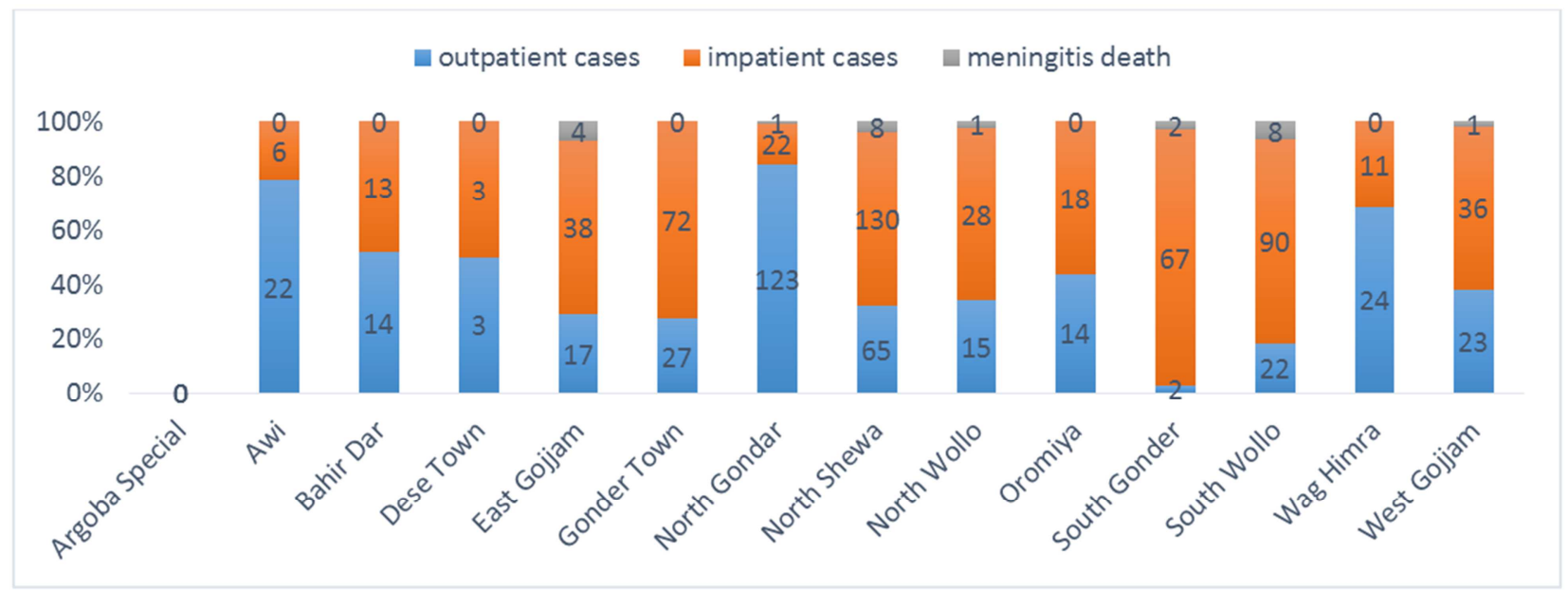

Figure 2. Impatient patients, outpatient patients and total deaths by zones of Amhara national regional state, Ethiopia 2015- 2019.

Regional patient fatality rate of the region is 2.80 and attack rate is 4.59 per 100,000 population. Patient fatality is high in East Gojjam (7.27\%) next to South wollo (7.92\%) while Attack rate is high in North Shewa $(9.52 / 100,000)$. Top ten disticts/health facilities reporting high patients of meningococcal meningitis were Gonder Town, Debrebrehan Hospital, Debretabor Hospital, West Armacho, Borena, Ataye Hospital, Legamibo, Enat Hospital, Finote Selam Hospital and Gandawuha respectively. Woldia and Enderta districts reports a single patient and a single death that make patient fatality rate $100 \%$. Argoba special district, Awi, Bahirdar liyu town, Desse, Waghimra, Oromia and Gondar town had not report any death and patient fatality rate is zero for those mentioned zones.

Meningococcal meningitis morbidity is high in North Gondar, South Wollo and North Shewa and mortality is high in North Shewa, South Wollo and East Gojjam respectively. The distribution is shown below in the regional map. Read color shows death and green color shows patients of meningococcal meningitis. The highest meningitis patient were reported from north Shewa in 2017, south wollo in 2018, north Shewa in 2016 and north Gondar in 2018. Argoba special district didn't report any meningococcal meningitis patient in any of the five year and Desse town reports negligible patients in any of the study period. 
Tesfahun Meshesha: Meningococcal Meningitis Data Analysis, Amhara National Regional State of Ethiopia, 2015-2019; a Descriptive Cross-Sectional Study

Table 1. Health indicators of meningococcal meningitis in Amhara national regional state, Ethiopia, 2015-2019 G.C.

\begin{tabular}{|c|c|c|c|c|c|}
\hline zone name & Total patients & Total deaths & Total population & $\mathrm{AR} / \mathbf{1 0 0 , 0 0 0}$ & CFR\% \\
\hline Total regional & 894 & 25 & $20,018,988$ & 4.59 & 2.80 \\
\hline Argoba Special district & 0 & 0 & 39,126 & 0 & 0 \\
\hline Awi & 28 & 0 & $1,143,639$ & 2.45 & 0 \\
\hline Bahir Dar Liyu Town & 27 & 0 & 313,415 & 8.61 & 0 \\
\hline Dese Town & 6 & 0 & - & - & 0 \\
\hline East Gojjam & 55 & 4 & $\overline{2,485,673}$ & 2.37 & 7.27 \\
\hline Gonder Town & 99 & 0 & - & - & 0 \\
\hline North Shewa & 195 & 8 & $2,131,857$ & 9.52 & 4.10 \\
\hline North Wollo & 43 & 1 & $1,733,616$ & 2.54 & 2.32 \\
\hline Oromiya & 32 & 0 & 529,995 & 6.04 & 0 \\
\hline South Gonder & 69 & 2 & $2,364,603$ & 3.00 & 2.90 \\
\hline South Wollo & 101 & 8 & $2,925,559$ & 3.73 & 7.92 \\
\hline Wag Himra & 35 & 0 & 487,324 & 7.18 & 0 \\
\hline West Gojjam & 59 & 1 & $2,422,296$ & 2.48 & 1.69 \\
\hline
\end{tabular}

$\mathrm{NB} . \mathrm{AR}=$ attack rate and $\mathrm{CFR}=$ patient fatality rate

Map. Amhara national regional state meningococal meningitis case distribution by woreda

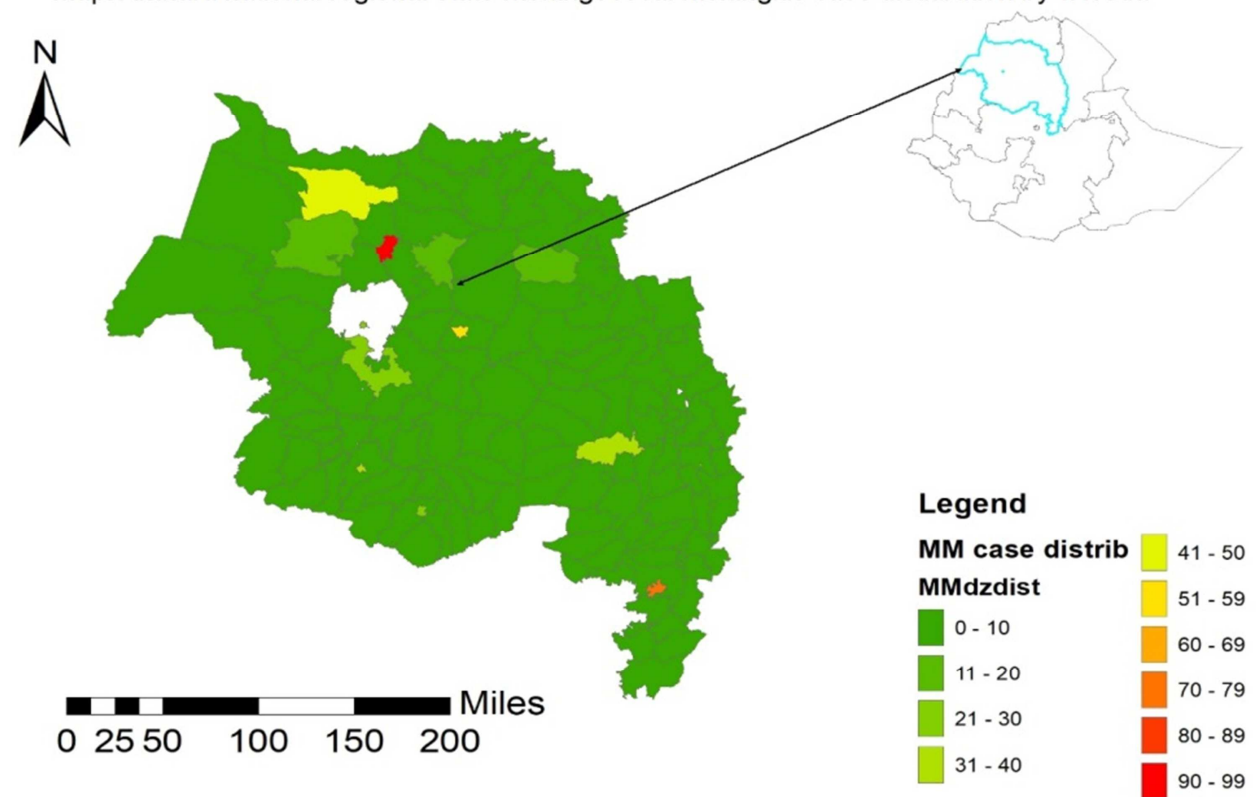

Figure 3. Distribution of meningococcal meningitis morbidity in districts of Amhara national regional state, Ethiopia 2015- 2019. Map source ESRI; Arc GIS version 10.4.1 and URL link https://www.esri.com/en-us/arcgis/products/arcgis-online/capabilities/make-maps

\subsection{Analysis by Time}

The overall trend of meningococcal meningitis in Amhara region is slightly increased from 2015- 2019 as shown in the graph bellow. Except in 2015 and 2017 the patient is below 200 even though there is ups and downs.

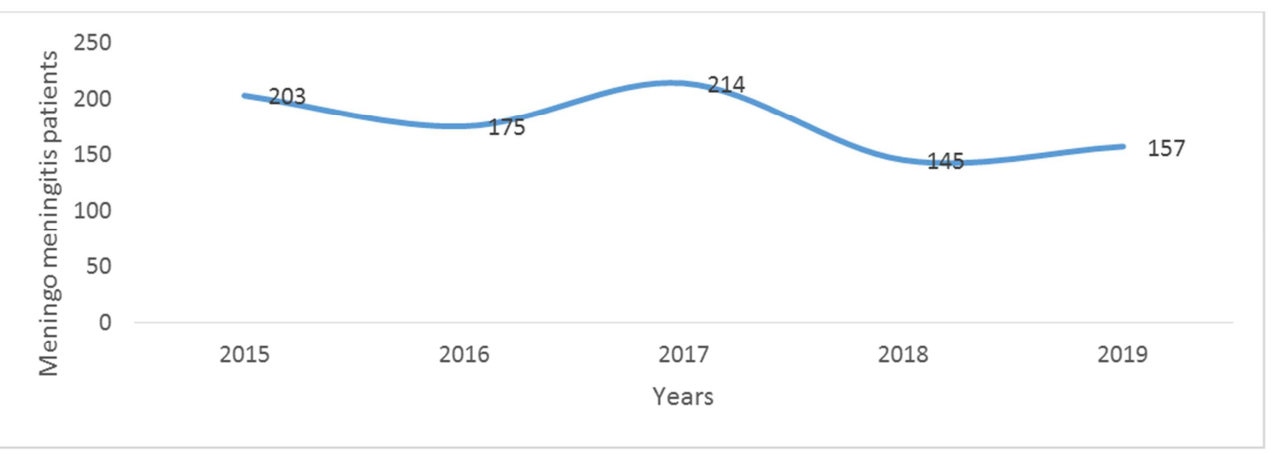

Figure 4. Trend of Meningococcal meningitis from 2015- 2019 in Amhara national regional state, Ethiopia. 
In epidemic week 38 of 2015 there was a meningococcal meningitis outbreak in north Gondar specifically in west Armachiho district. In this week about 48 patients reported but no prior or subsequent patients reported in the weekly surveillance data. In 2016 there were outbreaks of Meningococcal meningitis in South Wollo as communicated with the regional PHEM core process owners but the surveillance didn't detect the outbreak and didn't included in this study result. The figure revealed that two peaks were occurred during the five years, the first higher peak was observed in September, 2015 and the second peak was occurred during November, 2016.

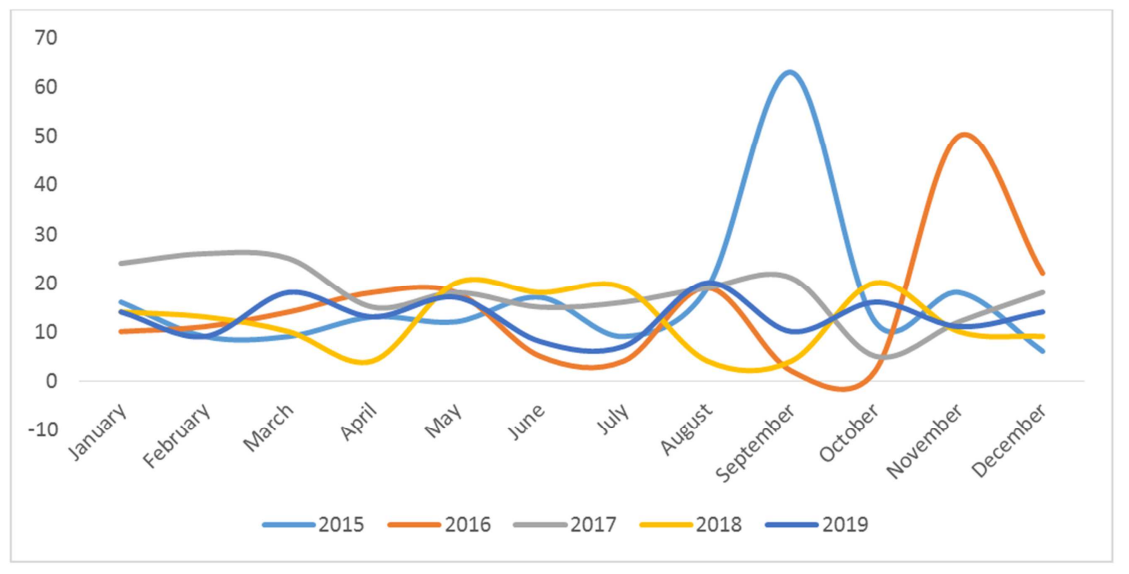

Figure 5. Trends of Meningitis Patients by Month and years, Amhara national regional state, Ethiopia, 2015- 2019.

\section{Discussion}

The analysis shows that there is a relationship between dry seasons and meningococcal meningitis case increment in the region. Regardless of the discrepancies of the number of patients in different zones and towns, meningococcal meningitis was remained seasonal with the highest number of patients during the dry season (December- March) and decreasing during the wet or rainy season. The result revealed that two gross peaks were occurred during the five years, even though the first highest peak was observed in September 2015 (during which most of the country is wet season and looks unusual, the second peak was occurred during the beginning of December in 2016 which is dry season in most parts of regional states of Ethiopia. This is parallel with the different scientific study conducted before regarding meningococcal meningitis and the relationship with seasonal variation (dry season). Several studies have investigated the relationship between climate and meningitis using different approaches $[16,17]$.

The other result found from the data analysis was, during the epi week 38 (dry season) in 2016 G.C There was a meningococcal meningitis outbreak in west Armachiho of North Gondar surpassing the threshold.

Comparing with different zonal reports, the highest incidence rate were recorded in North Shewa (9.52 $/ 100,000$ population) while the highest patient fatality rate were recorded in South Wollo (7.92 \%) followed by East Gojjam (7.27\%). The reason for the question "why meningococcal meningitis patient's distribution greatly affected by place?" could be multi factors which need farther study, but the most probable reason associated with this patient can be the level of timely intervention, vaccination coverage status: efficacy, migration, climate change and drought [18]. Meningococcal meningitis mortality is high in North Shewa, South Wollo and East Gojjam respectively. This result shows that patient management is poor in those high mortality areas like [19]. In another way high morbidity with low mortality like in North Gondar and North Shewa mean good patient management practice and laboratory capacity.

\section{Conclusion}

The trends of meningitis patient distribution within the five years were recorded the highest at the dry season of the years and progressively decrease at the wet or rainy season, this trend is exactly meeting with the various scientific literature. Meningococcal disease is highest in Amhara region with incidence rate of 4.59 per 100,000 populations and with $2.8 \%$ of Patient fatality rate during the five years. The highest Incidence rate patients during the five years was from North Shewa and Bahir Dar Liyu town with 9.52/100,000 and $8.61 / 100,000$ populations respectively, while the most affected zones were South Wollo, East Gojjam and North Shewa with CFR of $7.92 \%, 7.27 \%$ and $4.10 \%$ respectively.

\section{Recommendation}

1) Patient management practice should be improved in those with high burden of mortality.

2) Special emphasis to be given for Meningococcal meningitis especially during dry season when the disease is increasing.

3) Enhanced lab based surveillance should be improved and data quality at EPHI/PHEM should be assured and improved.

4) Different variables like age, sex and vaccination status 
of the patient should be included in the collected weekly surveillance data.

5) Laboratory based surveillance should be implemented to identify common serotypes of N. meningitides (The major pathogenic serogroups are A, B, C, W135, X and Y).

6) Further Research should be initiated based on primary data to solve unclear issues raise on the discussion.

\section{Author Contributions}

The author requests the data from Ethiopian Public Health Institute and made analysis and interpretation. The coauthor read and approved the final manuscript.

\section{Conflict of Interest Statement}

The authors declare that they have no competing interests.

\section{Availability of Data and Materials}

The data that support the findings of this study are available from Ethiopian Public Health Institute but are not publicly available. Data are however available from the authors upon reasonable request and with permission of Ethiopian Public Health Institute.

\section{Data Access}

A concept note requesting a 5 year meningitis data was submitted to the early warning and response case team of PHEM directorate and get access the data with a restriction of any description that identifies the personality of the study units will not be utilized. In addition to this, the analyst had traveled to Bahirdar (capital of Amhara national regional state) and collect supportive data's like line lists.

\section{Acknowledgements}

First of all I love to express my sincere and deepest appreciation to the Ethiopian Public Health Institute which provide me a five year meningitis data and St. Paul hospital millennium medical college. My special thank goes to Caroline furfur (IT expert at WHO) for her technical support. Last but not list I would say thank my wife Tigist Abera for her contribution in covering all routine life expenses while I am engaged in the data analysis.

\section{References}

[1] Haimanot RT, Caugant DA, Fekadu D, Bjune G, Belete B, Frøholm LO, et al. Characteristics of serogroup A Neisseria meningitides responsible for an epidemic in Ethiopia, 198889. Scand J Infect Dis. 1990.

[2] Mohammed I, Nasidi A, Alkali AS, Garbati MA, et el, severe epidemic of meningococcal meningitis in Nigeria, 1996. Trans R Soc Trop Med Hyg. 2000.
[3] Kaburi BB, Kubio C, Kenu E, et al. Evaluation of bacterial meningitis surveillance data of the northern region, Ghana, 2010-2015. The Pan African Medical Journal. 2017.

[4] Norheim G, Rosenqvist E, Aseffa A, Yassin MA, Mengistu G, Kassu A, et al. Characterization of Neisseria meningitides isolates from recent outbreaks in Ethiopia and comparison with those recovered during the epidemic of 1988 to 1989.

[5] Wude Mihret, Tsehaynesh Lema, Yared Merid, Afework Kassu... Surveillance of Bacterial Meningitis, Ethiopia, 20122013.

[6] Li Y, Yin Z, Shao Z, Li M, Liang X, Sandhu HS, Hadler SC, Li J, Sun Y, Li J, et al.; Acute Meningitis and Encephalitis Syndrome Study Group. Population based surveillance for bacterial meningitis in China September 2006-December 2009. Emerg Infect Dis 2014.

[7] Habte-Gabr E, Muhe L, Mamo M. Meningococcal meningitis in Ethiopia 1974-1983 and strategies of control. Ethiop J Health Dev. 1984; 1:47-63.

[8] Fitzwater SP, Chandran A, Santosham M, Johnson HL. The worldwide impact of the seven-valent pneumococcal conjugate vaccine. Pediatr Infect Dis J. 2012; 31 (5): 501-8.

[9] Emergency preparedness, response. Meningococcal disease: 2013 epidemic season in the African Meningitis Belt (Accessed: 12-07-2016).

[10] Rosenstein NE, Perkins BA, Stephens DS, Lefkowitz L, Cartter ML, Danila R, Cieslak P, Shutt KA, Popovic T, Schuchat A, et al. The changing epidemiology of meningococcal disease in the United States, 1992-1996. J Infect Dis. 1999.

[11] Greenwood BM. 1999 Manson lecture. Meningococcal meningitis in Africa. Trans. Roy. Soc.Trop. Med. Hyg. 93, 341-353. (doi:10.1016/S0035-9203(99)90106-2)

[12] The Ethiopian health and nutrition research institution Federal republic of Ethiopia national guideline on Meningococcal Amenities surveillance and outbreak management: first edition November 2013.

[13] Control of epidemic meningococcal disease. WHO practical guidlines.2nd edition.

[14] WHO Disease Outbreak Report, 17 August 2000Meningococcal disease in Ethiopia.

[15] Wude M, Tsehaynesh L, Yared M, Afework K, et el: Surveillance of Bacterial Meningitis, Ethiopia, 2012-2013, emerging infectious diseases journal, Volume 22, Number 1-2016.

[16] Mueller JE, Gessner BD. 2010 A hypothetica explanatory model for meningococcal meningitis in the African meningitis belt. Int. J. Infect. Dis. 14, 553-559. (doi: 10.1016/j.ijid.2009.08.013)

[17] Holliday, R. A, 2011. An Invitation to Health: Choosing to Change. J. Nutr.Educ. Behav. 43: 424.

[18] Leimkugel J, Hodgson A, Forgor AA, Pflu"ger V, Dangy J-P, Smith T, Achtman S, Gagneux S, Pluschke G. 2007 Clonal waves of Neisseria colonisation and disease in the African meningitisbelt: eight-year longitudinal study in northern Ghana. PLoS Med. 4, 10. (doi:10.1371/journal.pmed.0040101)

[19] Infection symptoms and air humidity with meningococcal carriage in Burkina Faso. Trop. Med. Int. Health 13, 15431552. (doi:10.1111/j.1365- 3156.2008.02165.x) 